\title{
La formación actual del médico: fortalezas y debilidades
}

\author{
X. Clèries, A. Martín-Zurro
}

\begin{abstract}
Las 'Jornadas estatales de estudio y debate sobre el futuro de la formación integral del médico ¿bioingeniería o medicina?' que se realizaron en Barcelona el 8 y 9 de febrero de 2008 fueron el marco para que un grupo de trabajo abordara el tema de las fortalezas y debilidades de la formación actual del médico. Las principales aportaciones se estructuraron en los tres niveles educativos siguientes: pregrado, posgrado y formación continuada. Se destacó la necesidad de una formación integral y psicosocial en la enseñanza de pregrado, la conveniencia de una estructuración troncal de la formación de posgrado para conseguir una mayor transversalidad de la competencia del médico y la contextualización de la formación continuada en el desarrollo profesional de cada médico. El predominio de los conocimientos biomédicos y tecnológicos en la formación actual del médico contrastan con las demandas de carácter psicosocial de una gran parte de la población en el sistema sanitario.
\end{abstract}

Palabras clave. Educación médica.

\section{The current training of doctors: strengths and weaknesses}

The 'National Conference for study and debate on the future of the integral training of doctors: bioengineering or medicine?' that were realized in Barcelona on February 8-9th, 2008 it was the context so that a work group was carried out on the topic about strengths and weaknesses of the current training of physicians. The main contributions were structured around three educational levels: undergraduate, postgraduate and continuing education. In the undergraduate level was emphasized the need to achieve an integral and psychosocial education. The postgraduate education should have a core subject in order to acquire a better global understanding of doctors' competences. Continuing medical education in the context of professional development of every physician. The predominance of the biomedical and technological knowledge in the current training of doctors contrasts with psychosocial demands of the population of healthcare system.

Key words. Medical education.

\section{Introducción}

El título del presente artículo dio nombre al grupo de trabajo que se llevó a cabo en el marco de las 'Jornadas estatales de estudio y debate sobre el futuro de la formación integral del médico ¿bioingeniería o medicina?' que se realizó en Barcelona a principios de febrero de 2008. La finalidad de estas jornadas era reflexionar sobre la realidad de la formación de los profesionales de la medicina en los países tecnológicamente desarrollados y sobre diferentes modelos, teorías y herramientas metodológicas. El grupo de trabajo estuvo formado por 30 profesionales de la medicina, de la psicología y del trabajo social, más una estudiante de medicina. Las aportaciones fueron recogidas y ordenadas por los coordinadores del grupo de trabajo para luego ser revertidas y ratificadas por todos los miembros del mismo grupo. Los resultados se estructuraron en tres niveles educativos: formación de pregrado, la formación de posgrado y la formación continuada. A continuación se especifican y desarrollan los principales contenidos de cada nivel educativo.
Institut d'Estudis de la Salut. Barcelona, España.

\section{Correspondencia} Xavier Clèries. Institut d'Estudis de la Salut. Roc Boronat, 51-85. E-08005 Barcelona.

Fax

+34935517519.

E-mail

fx.cleries@gencat.cat

Agradecimientos Los autores agradecen el apoyo recibido de J.L. Tizón y N. Daurella, coordinadores de las Jornadas Estatales de estudio y debate sobre el futuro de la formación integral del médico, así como la colaboración de los miembros del comité organizador y del comité científico. 


\section{Formación pregraduada del médico}

La identificación de los problemas de las facultades de medicina españolas fue clara y contundentemente señalada hace ya más de un decenio. Nuestras autoridades académicas han sido incapaces de introducir en los planes de estudio y en la organización y metodología de los procesos de enseñanza-aprendizaje y de evaluación, los cambios visualizados universalmente como imprescindibles para mejorar la calidad y pertinencia de la formación médica pregraduada [1]. A partir de este diagnóstico, compartido mayoritariamente por el grupo, se consideró que los objetivos de la formación pregraduada del médico deberían centrarse en la consecución de un profesional básico, adecuadamente preparado para iniciar un período de formación especializada. De manera especial, el perfil de este médico básico debería incidir en una orientación hacia una atención de salud integral e integrada, desde una perspectiva biopsicosocial de los procesos de atención, centrándose en las necesidades y expectativas de los pacientes y observando los problemas de salud de mayor relevancia en cada entorno. Para ello, el proceso de enseñanza-aprendizaje pregraduado debería asumir una formación teórica y práctica equilibrada y con base en la integración de los contenidos básicos y clínicos [2]; la tutorización activa y continuada del alumno en el seno de grupos reducidos, con un contacto precoz con las instituciones asistenciales y a partir del establecimiento de espacios de formación común, interprofesionales, y una exposición equilibrada a los paradigmas tecnológicos y de la atención personal, todo ello en el marco de una estructuración troncal inicial del currículo que ha de ir seguida de distintas líneas de formación diferenciadas.

Respecto a los contenidos de la formación pregraduada, se llegó al acuerdo de que deberían contemplarse de forma prioritaria los aspectos siguientes: comunicación y relación asistencial, favoreciendo la capacidad de observación, reflexión y autoconocimiento [3], atención primaria de salud [4], promoción de la salud y prevención de la enfermedad, bioética, antropología y sociología, e información sobre las denominadas medicinas complementarias. Por lo que concierne a la evaluación de la formación pregraduada, ésta debe garantizar, a través de distintos instrumentos y métodos, la competencia en términos de conocimiento, habilidades y actitudes del médico básico. En este sentido, es importante que se introduzcan métodos de evaluación que potencien la bidireccionalidad, la detección de necesidades de aprendizaje y la introducción de medidas de reorientación docente que permitan su abordaje.

Contextualmente, las recomendaciones del Espacio Europeo de Educación Superior, el llamado acuerdo de Bolonia, conforman una oportunidad para el cambio conceptual, de orientación, de organización, metodológico, de contenidos y de evaluación de los currículos formativos [5]. Aunque no hay que obviar las amenazas que también supone en cuanto a las resistencias que se pueden generar por los estamentos universitarios más anquilosados.

\section{Formación médica posgraduada}

Resulta innegable que el conocido sistema MIR constituyó en su momento una revolución para la formación de los médicos españoles, incluso hoy continúa siendo un potente instrumento para mejorar la competencia de los profesionales y la calidad del sistema sanitario [6]. No obstante, en el supuesto de que se introduzcan los cambios sustantivos y necesarios, que apuntamos previamente, en la formación pregraduada para garantizar la competencia del médico básico formado, se deberían reconsiderar diversos aspectos del sistema de formación posgraduada, entre ellos el examen de acceso, conocido como examen MIR.

Desde un punto de vista organizativo, la estructuración troncal de la formación es imprescindible para conseguir la transversalidad de la competencia de los médicos formados y debe facilitar una elección posterior más fundamentada de la especialidad. Pedagógicamente, el tutor, su formación, acreditación y reconocimiento deben ser elementos nucleares del sistema docente. En cuanto a la evaluación durante el proceso de formación, con elementos de carácter sumativo y formativo, debe permitir garantizar la competencia del médico especialista. La evaluación global del médico al finalizar su período formativo no debe consistir en una prueba o examen único sino en recopilar toda la información evaluativa acumulada durante la formación y complemen- 
tarla, cuando sea preciso, con otros elementos objetivos de comprobación.

\section{Formación continuada del médico}

Actualmente, la formación continuada del médico tiene que situarse conceptual y operativamente en el contexto más amplio del desarrollo profesional continuo individualizado (DPCi) [7], valorando la práctica profesional, la formación recibida, la docencia realizada y el trabajo asistencial y de investigación llevado a cabo. En este sentido, la estimación de la formación recibida debería contemplar las actividades acreditadas oficialmente, el número de créditos mínimos para conseguir un nivel determinado y las actividades relacionadas con la especialidad y la práctica propia del profesional. En definitiva, es necesario introducir mecanismos de garantía de la calidad, del aprovechamiento e impacto que tienen las actividades formativas sobre la competencia y actuaciones del profesional. Por otro lado, la recertificación periódica de los profesionales debe ir imponiéndose de forma decidida [8], condicionada a una accesibilidad suficiente a las actividades formativas y basada en métodos objetivos y que prioricen la ayuda y el apoyo de los profesionales sobre los elementos sancionadores.

Finalmente, y habida cuenta de que una parte importante de la formación continuada del médico es sufragada por la industria farmacéutica, hay que advertir y prevenir de los posibles sesgos de deformación y de los conflictos éticos en las intervenciones asistenciales de los profesionales [9].

\section{Consideraciones finales}

El enorme progreso en las ciencias biomédicas y en la tecnología ha alcanzado de lleno a la medicina en los países avanzados de Occidente. Los conocimientos biomédicos y tecnológicos copan actualmente el paradigma prevalente en la educación médica. Esta predominancia de lo biomédico y tecnológico convive paralelamente con un aumento sin parangón de las demandas de la población, la medicalización de la vida cotidiana y el incremento de las expectativas fantasiosas de los ciudadanos, entre otros aspectos. Muchos profesionales médicos reconocen que buena parte de la demanda que atienden no es pertinente. Frecuentemente, las razones que llevan a la población a la consulta médica tienen que ver con el malestar, pocas veces patológico, resultante de los avatares de la vida cotidiana. Así, por ejemplo, la ansiedad y la tristeza, procesos normales y necesarios para afrontar los problemas existenciales, suelen ser tratadas con ansiolíticos y antidepresivos, de manera que dificultan que la persona adopte un papel responsable y activo ante su propia problemática.

La formación actual del médico debería contemplar también, además de los aspectos biomédicos y tecnológicos, los conocimientos acerca de cómo el ser humano afronta su malestar y sus enfermedades. Todo ello en aras de una mayor pertinencia, efectividad, eficiencia y equidad del sistema sanitario.

\section{Conceptos básicos}

- La enseñanza de pregrado de medicina debería orientarse hacia una atención de salud integral e integrada, desde una perspectiva biopsicosocial de los procesos de atención, centrándose en las necesidades y expectativas de los pacientes y observando los problemas de salud de mayor relevancia en cada entorno.

- La evaluación global del médico especialista debe agrupar toda la información valorativa acumulada durante la formación.

- La formación continuada del médico se debe situar conceptual y operativamente en el desarrollo profesional continuo individualizado (DPCi), con la valoración de la práctica profesional, la formación recibida, la docencia realizada y el trabajo asistencial y de investigación llevado a cabo.

- La formación actual del médico debería integrar, además de los aspectos biomédicos y tecnológicos, los conocimientos acerca de cómo el ser humano afronta su malestar y sus enfermedades. 


\section{Bibliografía}

1. Rozman C. La educación médica en el umbral del siglo Xxi. Med Clin (Barc) 1997; 108: 582-6.

2. Rodés J, Trilla A. Fórmulas para la integración de la formación básica y clínica en medicina. Med Clin (Barc) 1999; 379-82.

3. Clèries X. La comunicación. Una competencia esencial para los profesionales. Barcelona: Masson-Elsevier; 2006.

4. Martín-Zurro A. Algunos aspectos esenciales de la medicina de familia. In Grupo de trabajo de pregrado semFYC, eds. La medicina de familia como área de conocimiento. Series Documentos semFYC, n. ${ }^{\circ} 10$. Barcelona: semFYC; 1998; 9-12.
5. World Federation for Medical Education, Association for Medical Education in Europe. The Bologna process and medical education. URL: http://www.educmed. net/pdf/documentos/bolonia/procesoBolonia.pdf [10.06.2008].

6. Martín-Zurro A, Nadal J. La reforma del sistema MIR. El País, 2007. URL: http://www.elpais.com/articulo/salud/ reforma/sistema/MIR [10.06.2008].

7. Martín-Zurro A, Gual A. Desarrollo profesional continuo individual vs formación continuada. Educ Med 2005; 8: 5-6.

8. Pardell H. ¿Es oportuno introducir la recertificación de los médicos en España? Med Clin (Barc) 2005; 124: 344-7.

9. Rubio ML, Cordón F. Relación con la industria farmacéutica: ¿un dilema ético? Aten Primaria 2000; 25: 135-6. 